\title{
ANÁliSiS DE LA SITUACIÓN DE LA TRADUCCIÓN Y LA INTERPRETACIÓN EN EL ÁMBITO JUDICIAL EN ESPAÑA EN CASOS ESPECÍFICOS DE VIOLENCIA DE GÉNERO
}

\author{
Esperanza Mojica López \\ University of Alcalá, Spain \\ espexen@gmail.com
}

\begin{abstract}
Resumen: Las diferencias culturales y lingüísticas son algunos de los principales obstáculos a los que tienen que enfrentarse los/as inmigrantes en el marco de la realidad multicultural y plurilingüe en la que vivimos. En este punto aparece la figura del/la intérprete o mediador intercultural que interviene para que pueda darse una comunicación efectiva. Este trabajo de investigación se centra en los recursos de asistencia lingüística que se ofrecen a las mujeres extranjeras víctimas de violencia de género que han decidido iniciar un proceso judicial.

El objetivo principal de esta investigación es analizar el estado de la cuestión de la comunicación con usuarias extranjeras víctimas de violencia de género en el ámbito judicial a través de intérpretes y la evaluación de la protección del derecho al acceso igualitario a la justicia y a la red de asistencia integral, con el fin de dar pautas de mejora o creación de un servicio de interpretación de calidad en los juzgados y tribunales españoles, con intérpretes especializados en género para los casos de violencia contra las mujeres.
\end{abstract}

Palabras clave: Interpretación, comunicación, interpretación jurídica/judicial, violencia de género, TISP y género.

\begin{abstract}
Cultural and linguistic differences are some of the main barriers foreigners have to face within the multicultural and multilingual environment we live in. At this point the role of the interpreter or intercultural mediator appears, as he/she acts to facilitate communication. This research focuses on the language assistance resources that are provided to women who suffer from gender-based violence and have decided to start legal proceedings.

The aim of this research is to analyse the current situation surrounding communication in legal settings with foreign women who are victims of gender-based violence. I will also assess the protection of the right to equal access to justice and to the complete network of assistance at their disposal, in order to establish recommendations to improve and/or create a high-quality interpreting service in Spanish Courts that provides trained interpreters specialised in gender for cases of violence against women.
\end{abstract}

Key words: Interpretation, communication, legal interpreting, gender-based violence, public service interpreting and gender.

\section{Introducción}

La sociedad multicultural y plurilingüe en la que vivimos lleva consigo la existencia de una serie de barreras que hay que eliminar para que se dé una convivencia intercultural y que así el proceso de integración de la población extranjera en la sociedad de llegada sea lo más natural posible. Las diferencias culturales y lingüísticas son algunos de los principales obstáculos a los que tienen que enfrentarse los/as inmigrantes. Pero esta población no hispanohablante es usuaria también de los servicios públicos. Por lo tanto, cuando las barreras lingüísticas impiden la comunicación, tendrán que desarrollarse soluciones que permitan acceder a los servicios independientemente de que haya un desconocimiento del idioma. En este punto aparece la figura del/la intérprete o mediador intercultural que interviene para que pueda darse una comunicación efectiva. 
De este modo nos encontramos con un triángulo que incluye al proveedor de servicios, al usuario, y al intérprete. En esta ocasión me centraré en uno de los vértices de este triángulo, el intérprete, y concretamente en los recursos de asistencia lingüística que se ofrecen para las mujeres extranjeras víctimas de violencia de género.

Según la Macroencuesta sobre Violencia de Género de 2011 realizada por el Centro de Investigaciones Sociológicas (CIS) en colaboración con el Gobierno "la prevalencia de la violencia de género entre las mujeres extranjeras duplica la de las mujeres españolas". Las barreras lingüísticas y culturales, sumadas a las desventajas de partida, aumentan la vulnerabilidad y desprotección de este colectivo (Amnistía Internacional, 2007: 11), donde se observa una triple discriminación: como mujer, como migrante y como víctima de violencia de género (Vela Díaz, 2012: 2). De este modo, lo que me ha llevado a querer investigar sobre este tema han sido los siguientes aspectos:

1. posibilidad de que las barreras lingüísticas deriven en una vulneración de los derechos de las usuarias migrantes no hispanohablantes que son víctimas de violencia de género.

2. posibilidad de explorar cómo podemos contribuir como profesionales de la comunicación a erradicar la violencia machista.

El presente estudio deriva del Trabajo Fin de Máster denominado "Interpretación en el ámbito judicial en casos de violencia de género" (Mojica-López, 2013) y constituye una primera aproximación a la realidad de la TISP en casos específicos de violencia de género, que pretende ampliarse mediante la elaboración de una Tesis Doctoral que abarcará datos más concretos y utilizará los resultados de otros estudios que se están realizando actualmente en esta materia.

\section{Marco teórico}

En los años noventa las movilizaciones feministas se encargaron de identificar, visibilizar y criminalizar la violencia machista, elevando el problema a un compromiso social. Estas luchas políticas y sociales que se desarrollaron a finales del siglo $\mathrm{XX}$, junto con un reposicionamiento de los medios de comunicación y un aumento de la conciencia social para erradicar la violencia de género, tuvieron influencia en las instituciones y los sectores jurídicos, quienes implantaron medidas para combatir esta violencia específica que se genera de la desigualdad histórica entre hombres y mujeres (Osborne, 2009: 12).

La Organización de Naciones Unidas ha sido el organismo internacional que ha elaborado los instrumentos jurídicos de mayor autoridad (ej.- Declaración de las Naciones Unidas sobre la eliminación de la violencia contra la mujer) y ha elevado el problema de la violencia ejercida contra las mujeres a un asunto de derechos humanos. La ONU ha instado a los Estados a aplicar leyes que pongan fin a la violencia de género $\mathrm{y}$, siguiendo la línea de estos instrumentos jurídicos, en 2004 entró en vigor la Ley Orgánica 1/2004 de Medidas de Protección Integral contra la Violencia de Género, también conocida como Ley Integral, lo que supuso todo un referente internacional, ofreciendo medidas de protección para las víctimas y creando los Juzgados de Violencia sobre la Mujer (JVM) para intentar conseguir la especialización del sector jurídico español en este ámbito.

A nivel europeo destacan los siguientes instrumentos jurídicos: La Directiva 2012/29/UE del Parlamento Europeo y del Consejo de 25 de octubre de 2012 por la que se establecen normas mínimas sobre los derechos, el apoyo y la protección de las víctimas de delitos, y por la que se sustituye la Decisión marco 2001/220/JAI del Consejo; y el Convenio del Consejo de 
Europa para prevenir y combatir la violencia contra la mujer y la violencia doméstica (también conocido como Convenio de Estambul).

Asimismo, el 5 de marzo de 2014 se han publicado en Viena los resultados del macroestudio sobre violencia de género en Europa. Este estudio, elaborado por la FRA (Agencia de los Derechos Fundamentales de la UE), y de escala de la UE, responde a una petición de datos sobre la violencia contra las mujeres realizada por el Parlamento Europeo, que el Consejo de la UE reiteró en sus conclusiones sobre la erradicación de la violencia contra las mujeres en la UE. Según los datos de este macroestudio, más de una de cada cinco mujeres $(22 \%)$ respondió a los entrevistadores que había sufrido esa violencia física y/o sexual por parte de una pareja o expareja. Por otra parte, a pesar de los instrumentos jurídicos y medidas aprobadas recientemente para erradicar la violencia de género, la mayoría de las mujeres víctimas de la violencia de género no recurren al sistema judicial ni a otros servicios, lo que pone de manifiesto que las necesidades y los derechos de muchas mujeres en la UE no se abordan en la práctica actualmente (FRA, 2013).

En cuanto a regulaciones específicas en materia de traducción en interpretación para las víctimas de violencia de género, en el artículo 32 (punto 4) de la Ley Integral, relativo a los planes de colaboración, se contempla a las mujeres migrantes como un colectivo especialmente vulnerable y que puede tener un mayor riesgo de sufrir violencia de género. Del mismo modo, en su artículo 18 (punto 3) se regula el derecho a la información para todas las víctimas de violencia de género, lo que implicaría la existencia de un servicio de traducción e interpretación para aquellas mujeres no hispanohablantes. La Directiva europea mencionada anteriormente incluye específicamente en su artículo 7 el derecho a traducción e interpretación y se recoge el hecho de que:

\footnotetext{
No se puede hacer justicia si no se permite a las víctimas explicar las circunstancias del delito y aportar pruebas de forma comprensible para las autoridades competentes. (...) Por lo tanto, siempre debe proporcionarse a la víctima un servicio de interpretación gratuito. (Diario Oficial de la Unión Europea, 2012: 60).
}

Sin embargo, existen casos documentados en los que el ejercicio de este derecho no se ha garantizado debido a las barreras lingüísticas (Amnistía Internacional, 2012: 7-8).

Existen dos figuras oficiales, la del traductor e intérprete jurado y la del traductor e intérprete del Ministerio de Justicia. Sin embargo, estos recursos no son suficientes para abarcar la gran demanda existente y, en la mayoría de los casos, se solicitan intérpretes a través de la empresa adjudicataria (MAEC, 2012: 25). La figura del traductor e intérprete de los servicios públicos no está reconocida y prueba de ello es la falta de regulación y profesionalización. Además, existe una falta de profesionalización y regulación al no contar con "colegios profesionales con sistemas coherentes de selección, formación, evaluación y acreditación" (Campos-López: 2005, 3). Existen asociaciones de traductores e intérpretes que intentan suplir esta carencia, pero lo cierto que "no son figuras profesionales reconocidas como los médicos o abogados" (Campos-López: 2005, 3).

\footnotetext{
La carencia de regulación mediante una licencia estatal u otras credenciales reconocidas nacional o internacionalmente significa que muchas personas sin formación profesional ni certificación alguna pueden ejercer el oficio de traductor o intérprete y ocupar puestos que, en mejores circunstancias, estarían destinados a los que sí han recibido una formación y tienen credenciales cualificado. Esto le resta valor a los estudios y credenciales profesionales y redunda ulteriormente en detrimento del cliente y de la profesión (MAEC, 2012: 6).
}

Existen numerosos instrumentos jurídicos a nivel nacional que recogen este derecho, pero muchos de ellos están obsoletos y permiten que pueda ejercer como intérprete cualquier persona conocedora de la lengua aunque no cuente con la formación o titulación necesaria 
(Gascón: 2010). A nivel internacional, el instrumento jurídico de mayor autoridad en este sentido es la Directiva 2010/64/EU, cuya transposición se ha hecho efectiva en octubre de 2013. Sin embargo, la falta de recursos económicos, teniendo en cuenta el contexto de crisis que vive el país y los consecuentes recortes que está realizando la Administración desemboca en una mayor externalización de los servicios de traducción e interpretación. En este caso la Administración fija las tarifas máximas y la empresa adjudicataria paga al intérprete (GarcíaNichols, 2013: 40). Con este tipo de contratación, la Administración se desentiende de este servicio y se ahorra la gestión de los mismos:

\begin{abstract}
La Administración de Justicia se ha desentendido del servicio de traducción e interpretación en muchos juzgados y ha dejado de ser el proveedor y organizador de dicha prestación. Por lo tanto, lejos de abordar el problema con firmeza, creando una estructura sólida y garantista, se ha preferido externalizar los servicios de traducción e interpretación y contratar a empresas privadas cuyo servicio ha quedado en entredicho en reiteradas ocasiones (Gascón, 2011: 32).
\end{abstract}

Sin embargo, se observa que están habiendo avances en la regularización y profesionalización de la TISP, como es la transposición de la mencionada Directiva europea, la publicación del Libro Blanco de la Traducción e Interpretación Institucional en España o la creación en 2009 de EULITA (European Union Legal Interpreters Association). La interpretación en el ámbito de la violencia de género es uno de esos campos de estudio específicos que están por explorar. Se están desarrollando medidas en este ámbito para cubrir las necesidades evidentes, como puede ser el proyecto "Speak Out for Support (SOS-VICS)" cuyo objetivo es mejorar la formación de intérpretes en el ámbito de la violencia de género, así como el estudio llevado a cabo por la Unidad Orgánica de Violencia de Género en la Red de Intervención Especializada en Violencia de Género en la Isla de Tenerife para evaluar la mediación lingüística en este tipo de casos o la "Jornada Formativa para Intérpretes de los Juzgados de la Comunidad de Madrid" (9 de octubre de 2013), organizada por la Dirección General de la Mujer².

\title{
2.1. Perfil y situación socioeconómica de las mujeres extranjeras residentes en España
}

La Declaración de las Naciones Unidas sobre la eliminación de la violencia contra la mujer hace referencia a las mujeres que sufren violencia de género y que son especialmente vulnerables (párrafo 1) como pueden ser las mujeres migrantes, y es que aunque la violencia machista afecta a todas las mujeres, independientemente de su edad, clase social, lugar de origen, etc., es necesario recalcar que, por ejemplo en España, la prevalencia de la violencia de género entre las mujeres extranjeras duplica a la de las mujeres españolas, de acuerdo con la Macroencuesta sobre Violencia de Género de 2011 realizada por el Centro de Investigaciones Sociológicas (CIS) en colaboración con el Gobierno.

Según estimaciones de la ONU, desde la década de los 60, el número de migraciones internacionales se ha duplicado, llegando a alcanzar una cifra de 190 millones de personas, el 3\% de la población mundial (United Nations, 2009). Dentro del marco de las migraciones internacionales, las mujeres representan más del 50\% en los movimientos migratorios procedentes de Europa, Oceanía y de la antigua Unión Soviética; en torno al $50 \%$ para América Latina, Caribe y América del Norte; y son minoría en los desplazamientos de África y Asia (Morrison, Schiff y Sjöblom, 2007). Según los datos de Censos de Población y

1 SPEAK OUT FOR SUPPORT (SOS-VIC) (JUST/2011/JPEN/2912): financiado por la Comisión Europea, Dirección General de Justicia (CÓDIGO DE REFERENCIA 400000 2912). Disponible en: http://cuautla.uvigo.es/sos-vics/.

Programa disponible en: http://cuautla.uvigo.es/sos-vics/entradas/veruno.php?id=109 
Viviendas 2011 (Instituto Nacional de Estadística), del total de extranjero residentes en España (5.252.473), 2.731.917 son hombres y 2.520.556 mujeres.

Como apunta Molpeceres Álvarez (2012: 2) "el peso y la cualidad de la participación de las mujeres en los flujos migratorios, no sería relevante si, como en el resto de dimensiones sociales, tales flujos no estuvieran influenciados y explicados por las relaciones de género". Las relaciones de género afectan a la hora de explicar quiénes y por qué razones emigran, cómo se toma la decisión de hacerlo, así como las consecuencias sobre las propias personas migrantes y los impactos tanto en las comunidades de origen y de destino (Jolly y Reeves, 2005).

Por lo que se refiere a feminicidios, a fecha de 19 de marzo de 2014, del número total de víctimas mortales por violencia de género (17), 6 eran extranjeras, lo que supone un 35,3\% del total (MSSSI, 2014).

Como señala la jurista e investigadora María Naredo Molero (2013) en la ponencia "La actuación profesional de los/as intérpretes en la atención a víctimas de violencia de género", las mujeres extranjeras que sufren este tipo de violencia suelen ser mujeres migrantes que han llegado en busca de una vida mejor y que cuentan con unas desventajas de partida como son el desarraigo de su país de origen, ausencia de una red de apoyo, el desconocimiento de las normas y la justicia y, en muchos casos, el idioma. Estas desventajas fomentan la dependencia económica de sus parejas, el aislamiento y el maltrato. En este momento aparece la figura del intérprete de los servicios públicos o mediador/a intercultural que elimina las barreras lingüísticas y culturales que existen entre las mujeres y los proveedores de servicios. Estos datos sobre el perfil de las mujeres extranjeras víctimas de violencia de género son necesarios para poder desarrollar propuestas de mejora de los servicios lingüísticos que asisten a estas mujeres, ya que en una situación interpretativa influyen muchos más factores además de las diferencias lingüísticas.

\section{Hipótesis de trabajo y objetivos}

La hipótesis que se plantea es la escasez de recursos y la falta de regulación y profesionalización que existe en torno a la asistencia lingüística destinada a las mujeres extranjeras que sufren violencia de género. Además, con esta investigación busco probar que la interpretación en casos de violencia de género es un tipo de interpretación especializada con una serie de características diferenciadas del resto de situaciones y contextos interpretativos que requiere tener una formación mínima en género y sobre la atención y el trato a las víctimas. Se entiende "género" como una categoría de análisis que permite entender las diferentes construcciones socioculturales de identidades, subjetividades, pautas de comportamiento o de relación...tanto para hombres como para mujeres, los diferentes roles atribuidos a unas u otros, los cuales no guardan relación con los atributos biológicos sexuados sino con las consecuencias socioculturales asociadas a estos, con un resultado de relaciones jerarquizadas, en las que las mujeres están subordinadas, real y simbólicamente, a los varones ${ }^{3}$.

Para probar esta hipótesis de trabajo se plantean los siguientes objetivos:

1. Valoración del estado de la cuestión: mujeres que no comparten la lengua y sufren violencia de género.

\footnotetext{
3 Guía de criterios de actuación judicial frente a la Violencia de Género del Consejo General del Poder Judicial (2013).
} 
2. Evaluación de la protección del derecho a asistencia jurídica igualitaria y a la red de asistencia integral para mujeres maltratadas cuando existen barreras linguísticas.

3. Extracción de terminología utilizada en materia de violencia de género para la posterior elaboración de un glosario de términos relevantes que sea de utilidad para los y las intérpretes.

4. Creación de propuestas para mejorar la asistencia lingüística a mujeres que sufren violencia de género.

5. Contribución a la concienciación sobre la importancia de eliminar las barreras lingüísticas y la necesidad de sustituir el actual modelo de subcontratación por la creación de un registro de intérpretes debidamente acreditados y con una formación especializada.

\section{Metodología}

La perspectiva metodológica es cuantitativa y cualitativa, lo que me ha permitido enfocar y guiar la investigación, determinar las variables y profundizar en los datos que se extraigan de los instrumentos de recopilación de información cuantitativa. De este modo, el tipo de investigación consiste en una combinación de ambas técnicas de investigación.

El análisis de los datos se ha realizado a partir de un corpus compuesto por encuestas y entrevistas a proveedores de servicios que trabajan con mujeres extranjeras que han iniciado un proceso judicial por violencia de género; informes generados a partir de la observación etnográfica de un juicio por violencia de género en el que la usuaria era extranjera y de una visita al centro penitenciario de mujeres Alcalá Meco I; y un caso judicial por agresión sexual y de otros documentos (cédulas de citación) que permitan extraer terminología jurídica propia de este ámbito específico para la posterior elaboración de un pequeño glosario bilingüe (español-inglés) que sea de utilidad para traductores e intérpretes, proveedores de servicios y usuarias extranjeras.

\section{Análisis de resultados}

El presente estudio se centra en el ámbito judicial, en el derecho a contar con un proceso judicial con las debidas garantías, en casos específicos de violencia de género donde las víctimas son mujeres extranjeras no hispanohablantes. Si no se facilitan los recursos necesarios, en este caso intérpretes de calidad con formación especializada, podríamos encontrarnos ante una discriminación ejercida por parte del Estado que desemboca en una vulneración de derechos, ya que no se garantiza al acceso igualitario a la justicia.

Los datos extraídos del corpus elaborado a partir de instrumentos de recopilación de información han sido muy útiles para conocer el estado de la cuestión y saber qué necesidades tienen las mujeres y los/as proveedores/as de servicios. El tamaño del corpus es reducido, ya que el objetivo de este estudio es hacer una aproximación a la situación de la interpretación en el caso de la violencia de género, pero la limitación de tiempo y espacio, unido al colapso de los juzgados y a la dificultad de obtener entrevistas con mujeres, impide, por el momento, profundizar más allá en la investigación.

\subsection{Análisis terminológico}

El caso judicial y las cédulas de citación han sido de gran utilidad para extraer terminología y fraseología propia del ámbito de la violencia contra las mujeres para la posterior elaboración 
del glosario. Además, demuestran el estilo arcaizante, críptico y amenazador del lenguaje jurídico patente en las cédulas, así como la cantidad de términos con conceptos indeterminados que aparecen en el lenguaje jurídico. El/la intérprete debe tener presente todas estas particularidades para transmitir el sentido a la otra lengua con precisión.

Tanto la legislación española como los instrumentos jurídicos de la Unión Europea han recogido la definición establecida en el artículo 1 de la Declaración de Naciones Unidas sobre la Eliminación de la Violencia sobre la Mujer. El artículo 2 se centra en delimitar el significado de violencia física, sexual y psicológica. Lo interesante de este artículo es que para definir los tipos de violencia se basa en el espacio en el que se producen: ámbito familiar, comunidad en general y aquella perpetrada o tolerada por el Estado, donde quiera que ocurra.

La terminología en el ámbito jurídico es de gran importancia y la delimitación de los significados esencial para la correcta aplicación de las leyes y, por supuesto, para la actuación de los intérpretes. Los conocimientos sobre la terminología que se utiliza en el ámbito de la violencia de género deben ser un aspecto crucial en la formación y especialización del/la intérprete. La precisión en la utilización de las unidades terminológicas, fraseología, etc. propia de este campo de especialidad es esencial para desarrollar la labor interpretativa de forma efectiva y profesional. El lenguaje jurídico es de por sí un ámbito del saber que necesita ser estudiado y analizado detalladamente para poder utilizarlo correctamente. Los traductores e intérpretes no son juristas y, por lo tanto, no se les puede exigir que actúen como tal. Sin embargo, son especialistas de la lengua y asimismo, deben contar con conocimientos necesarios relativos a la documentación para poder dominar el lenguaje con el que trabajan. Una herramienta muy útil puede ser un glosario con términos clave que ofrezcan definiciones claras para facilitar la labor de los/as intérpretes que trabajan con mujeres víctimas de violencia de género. En este trabajo de investigación tan solo se ofrece un esbozo que puede ampliarse posteriormente con más términos, aclaraciones, ejemplos de uso, etc.

\subsection{Encuestas}

Todas las informantes (tres psicólogas, una abogada y una agente de igualdad) de la encuesta sobre la comunicación con usuarias extranjeras víctimas de violencia contra las mujeres aseguran que a menudo se han encontrado en situaciones en las que no podían comunicarse con las usuarias que inician un proceso judicial por violencia de género debido a las barreras linguiísticas y que, por lo tanto, han tenido que recurrir a intérpretes o mediadores/as interlingüísticos. En la mayoría de los casos son las ONG las que ofrecen estos servicios a través de personal voluntario, lo que demuestra la falta de concienciación del Estado al ignorar el problema o delegar en otros organismos estos servicios. La utilización de intérpretes no formados puede acarrear muchos problemas relativos a la precisión, imparcialidad, fidelidad, profesionalidad, etc. al no contar con las estrategias necesarias para desempeñar esta labor. En muchos otros casos la situación es aún más grave, ya que son las propias usuarias las que buscan sus propios recursos para comunicarse con la ayuda de amigos, familiares e incluso hijos menores que dominan mejor el idioma. En general, lo datos demuestran la gran falta de regulación y los vacios existentes en torno a la asistencia lingüística y de calidad en los procesos judiciales.

En cuanto al perfil ideal de los/as intérpretes para este tipo de casos, las informantes responden de manera unánime que debe ser una intérprete (sujeto leído como mujer) quien actúe en este tipo de casos, ya que comparte una misma realidad social y se creará un ambiente de mayor confianza para la usuaria y será más fácil establecer la comunicación. En cuanto a la opinión sobre si la intérprete debería compartir la cultura de la usuaria, también se 
hace referencia al factor empático y a la importancia de dominar la cultura para poder acceder a la información que exceda el plano lingüístico, pero siempre evitando caer en generalizaciones y estereotipos y en el trato de la situación desde un punto de vista occidental y etnocéntrico. Prima siempre la individualidad de la persona. Otro dato importante es que todas prefieren que sea la misma intérprete la que intervenga en todas las sesiones de un mismo caso para que haya continuidad, sea más fácil establecer un vínculo de confianza con la usuaria, se ahorre tiempo y, sobre todo, se evite caer en la victimización secundaria que intensifique la situación de estrés de las mujeres.

\subsection{Consulta Instituto de la Mujer}

La respuesta a la consulta realizada al Instituto de la Mujer sobre la gestión de la comunicación con mujeres extranjeras demuestra también que existe una falta de regulación en torno a los servicios de asistencia lingüística. Existen recursos disponibles para casos de extrema urgencia pero no para el acceso a los servicios de información y atención integral.

\subsection{Entrevistas}

Las entrevistas realizadas a las trabajadoras sociales de la Dirección General de la Mujer (DGM) y del Servicio de Atención a la Mujer (SAM) arrojan datos similares. La primera informante asegura que se han recortado los pocos recursos con los que contaban y que literalmente "se las apañan como pueden" para comunicarse con las mujeres extranjeras víctimas de violencia de género que no hablan español. La trabajadora social del SAM aporta datos un poco más alentadores, ya que siempre cuentan con intérpretes que asistan a las usuarias. Sin embargo, estos profesionales se facilitan a través de la empresa adjudicataria y no existen pruebas de que se sigan rigurosos controles de calidad y que los profesionales cuenten con la formación necesaria para ejercer como intérpretes.

\subsection{Informes observación etnográfica}

Los informes extraídos de la observación etnográfica de un juicio por violencia de género en un Juzgado de Violencia sobre la Mujer de Madrid y de la visita al Centro Penitenciario de Alcalá-Meco Mujeres I también demuestran la falta de regulación en este sentido. Se ignora el problema y se deja prácticamente en manos de las usuarias la responsabilidad de eliminar estas barrearas comunicativas, creando una discriminación evidente y aumentando la vulnerabilidad de este colectivo. Todos estos datos ponen de manifiesto que se ha optado por el modelo puré ("melting pot") o modelo de asimilación en el que es el individuo el que debe cambiar y adaptarse a la sociedad de llegada, en vez de ofrecer un entorno apropiado y recursos de asistencia para facilitar el proceso de integración (Valero-Garcés, 2003: 89).

\section{Conclusiones y propuestas}

En primer lugar sería conveniente eliminar el actual modelo de subcontratación cuya prioridad es la rentabilidad económica y que trae consigo una serie de problemáticas, ya que tal como apunta Garrido y Plaza (2010: 123):

No existe un control, por parte de la Administración, sobre el cumplimiento de los pliegos de las licitaciones ni sobre la calidad del servicio suministrado, y han aparecido en la prensa numerosos casos de actuaciones de intérpretes que no tenían la calidad suficiente e incluso tenían antecedentes penales. (Garrido y Plaza, 2010: 123) 
Como proponen algunas asociaciones de traductores e intérpretes (APTIJ, 2012) y la propia CCDUTI (Del Pozo-Triviño, 2013: 123), lo ideal es crear un registro de traductores e intérpretes cualificados, debidamente acreditados y con experiencia profesional que sigan un estricto código de conducta.

En casos específicos de violencia de género, es recomendable que los traductores e intérpretes estén especializados en género y feminismos para poder comprender la multitud de aristas que confluyen en la violencia estructural ejercida contra las mujeres. Como recomienda el Informe Anual del Observatorio Estatal contra la violencia de género (2007: 194), es esencial la "formación en violencia de género de intérpretes y personas expertas en mediación intercultural". Además, teniendo en cuenta las necesidades manifestadas por las propias usuarias y por los/as proveedores/as de servicios entrevistadas y encuestadas para realizar este estudio, sería conveniente que estos profesionales fueran, en la medida de lo posible, mujeres, para evitar crear una situación hostil y aumentar la situación de estrés de la víctima. También es recomendable, según los datos extraídos, que las intérpretes sean de la misma cultura que la usuaria, o que por lo menos dominen ambas culturas, para poder descodificar los factores que entran en juego en la comunicación y que exceden el plano lingüístico.

Por último, considero que sería de gran utilidad que se elaborara una guía de buenas prácticas para las intérpretes y proveedores de servicios que ayude a delimitar las funciones de estas profesionales y establezca pautas de actuación para la multitud de situaciones particulares que se pueden dar en una sesión interpretativa.

Aunque parece que en el proceso penal está garantizado el derecho a ser asistida por un intérprete, no hay medidas que garanticen la calidad y la especialización en género de estos profesionales. Actualmente, parece que se está incrementando la concienciación sobre la importancia de la regulación de los servicios de traducción e interpretación en el ámbito judicial, tal y como demuestran las prevenciones de mejora del Consejo General del Poder Judicial, pero la falta de recursos económicos hace que se siga optando por la externalización de los servicios y la subcontratación, lo que desemboca en una falta de controles de calidad y en la precarización de la situación laboral de los/as profesionales lingüísticos.

En definitiva, sería conveniente que se llevara a cabo un proceso de regularización y profesionalización de los traductores e intérpretes de los servicios públicos $\mathrm{y}$, en el caso del ámbito del presente estudio, que se exigiera una formación específica y especialización en género para poder trabajar en el ámbito judicial con víctimas de violencia de género. Sin afán de extenderme mucho más en este sentido, considero que sin una base formativa en género y feminismos no es posible entender todos los factores que entran en juego en la violencia machista y la desigualdad de género. De este modo, con la formación adecuada los/as traductores e intérpretes podrían ser capaces de saber qué mecanismos ofrecer cómo profesionales de la comunicación y cómo actuar en estos casos, al ser parte del proceso de eliminación de la desigualdad de género.

En definitiva, tras esta aproximación a la situación de la traducción e interpretación en los servicios públicos y en especial a los casos de violencia de género mediados por intérpretes (y aunque la escasez de datos impida hacer generalizaciones), considero que sería necesario que se llevara a cabo en España un proceso de regulación profesional de los servicios de traducción e interpretación. Este es sin duda un proceso lento y que implica un desembolso económico. La esperanza en este sentido reside en la Directiva 2010/64/UE, que esperemos que suponga un punto de inflexión en la regulación profesional de los traductores e intérpretes en España. 


\section{Referencias bibliográficas}

APTIJ. 2012. Hacia un nuevo modelo de gestión de la traducción y la interpretación judicial. [Disponible en: http://www.aptij.es/img/doc/carta_vertice.pdf].

Amnistía Internacional. 2007. Más Riesgos y Menos Protección: mujeres inmigrantes en España frente a la Violencia de Género. [Disponible en: http://www.es.amnesty.org/uploads/media/Informe_Mas_riesgos_y_menos_proteccio n_231107.pdf].

Amnistía Internacional. 2012. ¿Qué Justicia Especializada? A siete años de la Ley Integral contra la Violencia de Género: obstáculos al acceso y obtención de justicia y protección. [Disponible en: https://doc.es.amnesty.org/cgibin/ai/BRSCGI/Que\%20justicia\%20especializada.informe\%202012?CMD=VEROBJ \&MLKOB $=32130865353$ ].

Campos López, J. 2005. Mediación intercultural y traducción/interpretación en los servicios públicos: el caso de la atención sanitaria a población inmigrante de origen magrebí. Fòrum de Recerca 10. Castellón: Universitat Jaume I.

C.G.P.J. 2012. Acuerdo adoptado por la Comisión de Modernización e Informática, de fecha 29 de mayo de 2012, en relación a las Prevenciones para mejorar la Traducción e Interpretación en Procedimientos Judiciales, aprobadas por la Sala del Gobierno en su Reunión de fecha 16 de abril de 2012.

[Disponible en: http://www.aptij.es/img/doc/prevenciones_matizadas.pdf].

Del Pozo-Triviño, M. 2013. El camino hacia la profesionalización de los intérpretes en los servicios públicos y asistenciales españoles en el siglo XXI. Cuadernos de Aldeeu, vol. 25, número especial. [Disponible en http://aldeeu.org/cuadernos/index.php/CALDEEEU/article/view/37/29].

España. Ley Orgánica 1/2004, de 28 de diciembre, de Medidas de Protección Integral contra la Violencia de Género. Boletín Oficial del Estado, 29 diciembre de 2004, núm. 313: 42166-42197. [Disponible en: https://www.boe.es/buscar/doc.php?id=BOE-A-200421760].

FRA. Agencia de los Derechos Fundamentales de la UE. 2013. Violencia de género contra las mujeres: una encuesta a escala de la UE. [Disponible en: http://fra.europa.eu/sites/default/files/fra-2014-vaw-survey-factsheet_es.pdf].

García Nichols. 2013. Interpretación en el ámbito judicial español. Disponible en Web: http://prezi.com/cy6cy67vkp9w/interpretacion-en-el-ambito-judicial-espanol/].

Garrido, R. y S. Plaza. 2010. Traducción contra la exclusión: los traductores e intérpretes de las Administraciones Públicas y el Libro Blanco de la traducción e interpretación institucional. Luis González Carmen las Heras (eds.) La traducción y la interpretación contra la exclusión social. [Disponible en: http://ec.europa.eu/spain/pdf/actas-jornada-traduccion-y-la-interpretacion-contra-laexclusion-social-1-octubre-2010_es.pdf].

Gascón, F. 2011. Una breve radiografía de la interpretación judicial en España. La Linterna del Traductor, núm. 6: 31-40. [Disponible en: http://www.lalinternadeltraductor.org/pdf/lalinterna_n6.pdf ].

Instituto Nacional de Estadística. 2011. Censos de Población y Viviendas 2011. [Disponible en:http://www.ine.es/jaxi/menu.dotype=pcaxis \&path=/t20/e244/avance/p01/\&file=pc axis\&L=0]

Jolly, S. y H. Reeves. 2005. Gender and migration. Overview Report. Brighton: Sussex University, Bridge. 
Ministerio de Asuntos Exteriores y Cooperación. 2012. Libro Blanco de la traducción y la interpretación institucional. [Disponible en:

http://ec.europa.eu/spain/pdf/libro_blanco_traduccion_es.pdf].

Ministerio de Sanidad, Servicios Sociales e Igualdad y el Centro de Investigaciones Sociológicos. 2012. Macroencuesta de Violencia de Género 2011. [Disponible en: http://www.observatorioviolencia.org/upload_images/File/DOC1329745747_macroen cuesta2011_principales_resultados-1.pdf ].

Ministerio de Sanidad, Servicios Sociales e Igualdad y el Centro de Investigaciones Sociológicos. 2014. Ficha estadística de víctimas mortales por Violencia de Género. Año 2014. [Disponible en: http://www.msssi.gob.es/ssi/violenciaGenero/portalEstadistico/docs/VMortales_2014 19_03.pdf ].

Ministerio de Sanidad, Servicios Sociales e Igualdad. 2012. Información estadística de violencia de género -Febrero 2012. [Disponible en: http://www.msssi.gob.es/ssi/violenciaGenero/portalEstadistico/InformesViolenciaGen ero/pdfs/Informe_VG_Febrero_2012.pdf ]

Mojica-López, E. 2013. Interpretación en el ámbito judicial en casos de violencia de género (2012-2013). [Trabajo fin de máster no publicado]. Universidad de Alcalá, Lenguas Modernas, Máster en Comunicación Intercultural, Traducción e Interpretación en los Servicios Públicos, España.

Molpeceres Álvarez, L. 2012. Situación laboral de las mujeres inmigrantes en España en Cuadernos de Relaciones Laborales Vol. 30, núm. 1: 91-113. Madrid: Universidad Complutense de Madrid. [Disponible en: http://revistas.ucm.es/index.php/CRLA/article/view/39116 ].

Morrison, A.R, Schiff, M. y M. Sjöblom. 2007. The international migration of women. New York: World Bank Publications.

Naredo-Molero, M. 2013. La actuación de los/las intérpretes en la atención a las víctimas de violencia de género [Archivo de Vídeo]. [Disponible en: https://www.youtube.com/watch?v=Lv1vg9Q5uZ4].

Observatorio Estatal de Violencia sobre la Mujer. 2007. Informe Anual del Observatorio Estatal de Violencia sobre la Mujer. Madrid: Ministerio de Trabajo y Asuntos Sociales. Secretaría General de Políticas de Igualdad. Delegación Especial del Gobierno contra la Violencia sobre la Mujer. [Disponible en: http://www.observatorioviolencia.org/upload_images/File/DOC1204104060_Informe AnualInternet.pdf]

Osborne, R. 2007. Apuntes sobre violencia de género. Barcelona: Bellaterra.

Unión Europea. Directiva 2012/29/UE del Parlamento Europeo y del Consejo de 25 de octubre de 2012 por la que se establecen normas mínimas sobre los derechos, el apoyo y la protección de las víctimas de delitos, y por la que se sustituye la Decisión marco 2001/220/JAI del Consejo. Diario Oficial de la Unión Europea L 315, de 14 de noviembre de 2012. 57-73. [Disponible http://eurlex.europa.eu/LexUriServ/LexUriServ.do?uri=OJ:L:2012:315:FULL:ES:PDF.00].

Unión Europea. 2011. Convenio del Consejo de Europa sobre prevención y lucha contra la violencia contra las mujeres y la violencia doméstica. Council of Europe Treaty Series No. 210. [Disponible en: http://www.coe.int/t/dghl/standardsetting/conventionviolence/convention/Convention\%20210\%20Spanish.pdf].

United Nations, Department of Economic and Social Affairs, Population division. 2009. International Migration Report 2006: A Global Assessment. [Disponible en: http://www.un.org/esa/population/publications/migration/WorldMigrationReport2009. pdf]. 
Valero Garcés, C. 2003. Soñé con una melodía y escuché voces dispersas. Barrearas en la comunicación interlinguiística en los centros de salud. En C. Valero Garcés (ed.) Discursos (Dis)Con/Cordantes: Modos y Formas de Comunicación y Convivencia. Alcalá de Henares: Servicio de Publicaciones. 89-109.

Vela Díaz, R. 2012. La incidencia de la Violencia de Género en las mujeres extranjeras y la importancia del trabajo como factor de integración social. $3^{\circ}$ Congreso para el Estudio de la Violencia contra las mujeres. Justicia y Seguridad. Nuevos Retos Granada, 26 y 27 de noviembre de 2013. [Disponible en: http://www.congresoestudioviolencia.com/2012/articulo11.php]. 